\title{
Studies on Heterosis in Cotton Interspecific Heterotic Group Hybrids (G.hirsutum X G.barbadense) for Seed Cotton Yield and Its Components
}

\author{
S. Rajeev*, S.S. Patil, S.M. Manjula, K.J. Pranesh, P. Srivalli and H.G. Kencharaddi \\ Department of Genetic and Plant Breeding, University of Agricultural Sciences, \\ Dharwad-580 005 (Karnataka), India \\ *Corresponding author
}

\begin{tabular}{|l|}
\hline K e y w o r d s \\
$\begin{array}{l}\text { Gossypium hirsutum } \mathrm{L} ., \\
\text { Hybrid breeding program, } \\
\text { Relative growth rate } \\
\text { (RGR), Randomized } \\
\text { block design (RBD) }\end{array}$ \\
\hline Article Info \\
\hline $\begin{array}{l}\text { Accepted: } \\
\text { 15 September } 2018 \\
\text { Available Online: } \\
\text { 10 October } 2018\end{array}$ \\
\hline
\end{tabular}

A B S T R A C T

\begin{abstract}
Line $\times$ tester analysis was undertaken to assess the magnitude of heterosis in 32 interspecific heterotic group hybrids (G. hirsutum x G. barbadense) of cotton for seed cotton yield and its components developed by crossing eight elite hirsutum lines with four elite barbadense testers during Kharif 2010-11 at Main Agricultural Research Station, Dharwad. The Line $\times$ tester analysis consisting 32 interspecific heterotic group hybrids along with checks (RAHB 87 and DCH 32). The results revealed that the variances among genotypes for all the characters were significant. The mean sum of squares for parents was significant for all the characters except for seed index. The hirsutum lines viz., RAH-16 and DHMS and barbadense lines viz., RAB-8 and DB 534 recorded highest mean seed cotton yield. Among hirsutum $\mathrm{x}$ barbadense crosses viz., the hybrids viz., DHMS $\times$ SNICB75-10 (110.2), DH2752 × DB534 (95.8), DHMS $\times$ DB534 (94.4), RAH-25-17 $\times$ DB534 (90.9) and DH2752 $\times$ SNICB75-10 (89.4) exhibited highest significant positive heterosis over commercial check DCH-32. These interspecific crosses have shown desirable heterosis for seed cotton yield along with other yield components which may be tested in large scale trial to confirm the superiority in heterosis. Based on the predicted double cross performance, the cross combination DHMS $\times$ SNICB75-10 and DH2752 $\times$ DB534 has recorded the highest mean seed cotton yield of $3287 \mathrm{~kg}$ per ha and was selected as diverse $F_{1}$ base populations for initiating next phase of reciprocal selection for combining ability.
\end{abstract}

\section{Introduction}

Commercial cultivation of intra hirsutum hybrids first introduced in India during 1960s rapidly increased production and productivity of cotton in India, also lead to acute shortage of ELS cotton in the country. Right at this juncture a naval concept of bringing together the fibre properties of barbadense and productivity features of hirsutum marked the beginning of the era of interspecific hybrids leading to boom in ELS cotton cultivation in India. Interspecific hybrids introduced during 1970s especially the prominent hybrids from UAS Dharwad namely Varalakshmi and DCH 32 were instrumental in saving from ELS shortage and very critical foreign exchange running to hundreds of crores of rupees (Yanal, 2014). Just in about 10-15 years the popularity of interspecific hybrids started 
diminishing and by mid-1980, the intra hirsutum hybrids again took upper hands and currently interspecific hybrid area is restricted to few districts of Madhya Pradesh, Maharashtra, Karnataka and Tamil Nadu. To possible to bring back glory of ELS era, there is an urgent need to frame research priorities on improving potentiality of barbadense varietal base and developing hybrid oriented populations based on them and utilizing them in deriving potential interspecific hybrids. To increase productivity ELS cotton emphasis is necessary to implement this programme in interspecific hybrids.

Realizing the need for developing potential interspecific ( $G$. hirsutum x G. barbadense) hybrids studies were initiated at UAS Dharwad to identify hirsutum and barbadense genotypes capable of giving potential interspecific hybrids.

Heterosis works as a basic tool for improvement of crops in form of $F_{1}$ and $F_{2}$ populations, and economic heterosis (over standard cultivar). Heterotic studies can provide basis for exploitation of valuable hybrid combinations in future breeding program. Heterotic groupis a predicted choice of most potential heterotic pairs from the all interspecific crosses attempted between the $G$. hirsutum and $G$. barbadense lines. The potentiality of the heterotic group is predicted based on the potentiality of the non- parental combinations involved in the group.

This procedure is based on the method of predicting double cross performance followed in crops like maize where the genetically diverse single crosses are identified to develop double cross combination (Jenkins, 1934). The main objective of this study to study the heterosis and per se performance of new cotton inter specific hybrids in respect of seed cotton yield and its attributing characters and identify the potential heterotic cross combination based on the predicted double cross combination for initiating the reciprocal recurrent selection for developing the hybrid oriented populations.

\section{Materials and Methods}

The plant materials used in the present study were obtained by line $\mathrm{x}$ tester crossing of eight hirsutum lines, DH2752, DHMS, RAH-13-86, RAH-16, RAH-25-17, RAH-370, RAH-5-10 and DH-37 with four barbadense testers DB534, SNICB75-10, RAB-4 and RAB-8. The Line $\times$ tester crosses involving parents (eight lines from hirsutum and 4 testers from barbadense group) and 32 interspecific hybrids $(G$. hirsutum $\times G$. barbadense) along with two checks (RAHB 87 and DCH 32) were evaluated along with commercial checks in Randomized Block Design (RBD) with two replications. Each entry was sown in 2 row plots spaced at $90 \mathrm{~cm}$ with recommended dose of fertilizer and seeds were sown on 21-62014, 2-3 seeds were dibbled per spot in each row and thinning was attended to retain one healthy plant per hill at 25 days after sowing. All the recommended package of practices were followed to rise healthy crop.

Samples containing 20 bolls were handharvested from each plot prior to picking. The days to 50 per cent flowering recorded by the number of days taken from the date of sowing to the date when the first flower opens in 50 per cent of the plants. The number of monopodia per plant are the number of branches on main stem which were lateral and axillary in position with vertical growth in acropetal succession was counted at maturity stage, avoiding small sprouts, but the number of sympodia per plant are branches which are extra-axillary in position and normally horizontal with zig -zag pattern of fruiting points were taken as sympodia. The number of such sympodia on main stem were counted at maturity stage. The boll samples were 
weighed to determine seed cotton weight per boll values, and ginned on a roller using laboratory gin for lint percentage $(100 \mathrm{x}$ lint weight/seed cotton weight) and 100-seed weight calculations (seed index). The ginned lint from each plot was weighed and divided by the number of plants within each plot to determine lint yield per plant. Five plants were selected randomly from each genotype to find the boll number per plant. The Microsoft Excel computer program was used to analyze the data. The line $\mathrm{x}$ tester mating design according to Singh and Heterosis expressed as per cent increase or decrease of $F_{1}$ hybrid over mid-parent (mid-parent heterosis), better parent (heterobeltiosis) and the best commercial check (standard heterosis) were computed for each character using the following formulae (Turner, 1953 and Hayes et al., 1955). Out of three checks, the mean performance of the best check in each character was considered to work out the standard heterosis.

Heterosis over mid parent (relative heterosis)

$$
=\frac{\overline{\mathrm{F}}_{1}-\overline{\mathrm{MP}}}{\overline{\mathrm{MP}}} \times 100
$$

Heterosis over check (standard heterosis) = $\overline{\mathrm{F}}_{1}-\overline{\mathrm{CC}}$

\section{$\widetilde{C C} \times 100$}

Where,

$\overline{F_{1}}=$ Mean performance of $F_{1}$

$\overline{\mathrm{MP}}=$ Mean mid-parental value $=(\overline{\mathrm{P} 1}+\overline{\mathrm{P} 2}) / 2$

$\overline{\mathrm{P} 1}=$ Mean performance of parent one

$\overline{\mathrm{P} 2}=$ Mean performance of parent two

$\overline{\mathrm{CC}}=$ Mean performance of the best $\mathrm{Bt}$ hybrids as commercial check

\section{Results and Discussion}

Line $\times$ tester study involving 12 parents (Eight hirsutum lines and four barbadense testers) and their 32 hybrids were evaluated with commercial checks for confirming potentiality of identified heterotic box. Analysis of variance was carried out for 13 seed cotton yield and its component traits using the data obtained from inter specific heterotic group line $\times$ tester study. ' $F$ ' test was carried out to examine the significance of variances. The values of mean sum of squares for 13 characters are presented in table 1. It was observed that the variances among genotypes for all the characters were significant. The mean sum of squares for parents was significant for all the characters except for seed index. The mean squares with respect to hybrids were significant for most of the characters except for SPAD meter reading, lint index, seed index which depicts considerable differences among the hybrids for those traits. Variance arising from interaction between hybrids and parents was significant for all the characters except ginning out turn, SPAD meter reading and inter branch distance. The differences due to replications were not significant for all the characters.

Per se performance of twelve parents involved in thirty two crosses $(8 \times 4$ Line $\times$ Tester $)$ for seventeen different quantitative along with fibre characters are presented in Table 1. Per se performance and heterosis values of single cross hybrids over mid parent and commercial check for thirteen different quantitative characters obtained for the thirty two hybrids are presented in Table $2 \mathrm{a}, 2 \mathrm{~b}$ and $2 \mathrm{c}$.

A comparison of the mean value of the parents and hybrids in respect of different characters revealed wide range for seed cotton yield among hirsutum lines $(1,248 \mathrm{~kg} / \mathrm{ha}$ to 1,733 $\mathrm{kg} / \mathrm{ha})$ and barbadense testers $(1,263 \mathrm{~kg} / \mathrm{ha}$ to $1,419 \mathrm{~kg} / \mathrm{ha}$ ), while among the crosses it 
varied from $1674(\mathrm{RAH}-5-10 \times$ SNICB75-10) to $3500 \mathrm{~kg}$ per ha $(\mathrm{DHMS} \times \mathrm{SNICB} 75-10)$ with an overall mean of $2460 \mathrm{~kg}$ per ha. The lowest value was observed in barbadense line RAB 4 and highest value observed in hirsutum line RAH 16. The mean value observed for barbadense lines and hirsutum lines was 1318 $\mathrm{kg} / \mathrm{ha}$ and $1472 \mathrm{~kg} / \mathrm{ha}$, respectively. Out of thirty two hybrids studied, twenty four crosses recorded significant mid parent heterosis in positive direction, while none of the crosses showed significant negative heterosis over mid parent and the range was from 7.3 (RAH$16 \times \mathrm{RAB} 8)$ to $122.9(\mathrm{RAH}-25-17 \times \mathrm{DB} 534)$ per cent with a mean of 64.9 per cent. The heterosis over commercial check ranged from $0.6(\mathrm{RAH}-5-10 \times$ SNICB75-10) to 110.2 $($ DHMS $\times$ SNICB75-10) with a mean of 47.8 per cent. Twenty one hybrids recorded significant positive heterosis over commercial check. The hybrids viz., DHMS $\times$ SNICB7510 (110.2), DH2752 × DB534 (95.8), DHMS $\times$ DB534 (94.4), RAH-25-17 × DB534 (90.9) and DH2752 $\times$ SNICB75-10 (89.4) recorded highest significant positive heterosis over commercial check with respect to seed cotton yield. Presence of heterosis over mid parent and commercial check was reported by Somashekhar (2006), Deepakbabu (2007), Ramakrishna (2008), Pranesh (2014) and Reddy (2015).

The range for lint yield in hirsutum lines $(688 \mathrm{~kg} / \mathrm{ha}$ to $1065 \mathrm{~kg} / \mathrm{ha})$ was higher than the barbadense lines $(574 \mathrm{~kg} / \mathrm{ha}$ to $709 \mathrm{~kg} / \mathrm{ha})$. The lowest and highest lint yield was observed in barbadense line SNICB 75-10 and hirsutum line RAH 16, respectively. The mean of this character was observed to be higher in hirsutum $(845 \mathrm{~kg} / \mathrm{ha})$ than the barbadense lines $(623 \mathrm{~kg} / \mathrm{ha})$. The lint yield among the crosses was found to be vary from 562 (DH-37 $\times$ SNICB75-10) to $1,300 \mathrm{~kg}$ per ha (DHMS $\times$ SNICB75-10) with an overall mean of $852 \mathrm{~kg}$ per ha. The hybrids viz., DHMS $\times$ SNICB75$10(1300 \mathrm{~kg} / \mathrm{ha}), \mathrm{DH} 2752 \times$ DB534 $(1157$ $\mathrm{kg} / \mathrm{ha}), \mathrm{RAH}-25-17 \times$ DB534 (1080 kg/ha), DH2752 $\times$ SNICB75-10 (1073 kg/ha) and DHMS $\times$ RAB8 $(1068 \mathrm{~kg} / \mathrm{ha})$ occupied top five positions with respect to lint yield. Out of thirty two hybrids studied, nine crosses recorded significant mid parent heterosis in positive direction, while none of the crosses showed significant negative heterosis over mid parent and the range was from -18.1 $(\mathrm{RAH}-16 \times \mathrm{RAB} 8)$ to $74.8(\mathrm{DHMS} \times$ SNICB75-10) per cent with a mean of 56.4 per cent. The heterosis over commercial check ranged from $3.0(\mathrm{DH}-37 \times$ SNICB75-10) to $138.5($ DHMS $\times$ SNICB75-10) with a mean of 56.4 per cent. Twenty six hybrids recorded significant positive heterosis over commercial check. Significant positive heterosis over mid parent was reported by Potdukhe (2002), Punitha and Ravikesavan (2004) and Saifullah et al., (2014). Heterosis over commercial check was reported by Neelima (2002), Maisuria et al., (2006), Tuteja et al., (2014) Pranesh (2014)and and Reddy (2015).

The range number of bolls per plant in hirsutum lines (20.6 to 36.0) was higher than the barbadense lines (24.5 to 30.6). The lowest and highest number of bolls per plant was observed in hirsutum lines of RAH 5-10 and DH2742, respectively. The mean of this character was higher in hirsutum (29.2) than the barbadense lines (27.7). The variation for this trait among the crosses was from 35.0 $($ RAH-5-10 × SNICB75-10) 59.7 (DHMS $\times$ DB534) with an overall mean of 44.20. The hybrids RAH-13-86 $\times$ RAB-4 (105.2 per cent) and RAH-5-10 $\times$ RAB-8 (91.0 per cent) showed the highest mid parent heterosis in positive direction. The mean heterosis was 56.90 per cent. Twenty nine crosses depicted significant positive heterosis over mid parent. The values of heterosis over commercial check varied from -8.40 (RAH-5-10 $\times$ SNICB75-10) to $56.30($ DHMS $\times$ DB534) with a mean of 15.7 per cent. Sixteen crosses recorded significant positive heterosis over 
commercial check. Prevalence of significant heterosis for this trait was in accordance with the studies of Kajjidoni (1982), Reddy (2001), Deepakbabu (2007) Ramakrishna (2008) and Tuteja et al., (2014) and Pranesh (2014).

There was considerable difference observed in the range for boll weight in hirsutum lines (3.6g to $4.6 \mathrm{~g}$ ) compared to barbadense lines (3.3g to $3.8 \mathrm{~g}$ ). The lowest value was observed in barbadense line RAB 8 line and highest value observed in hirsutum line DHMS. The mean for this trait was higher in hirsutums (4.1g) than the barbadense (3.1g). The mean value boll weight ranged from 4.00 (RAH-5$10 \times \mathrm{RAB} 4)$ to $7.20 \mathrm{~g}(\mathrm{DHMS} \times \mathrm{SNICB} 75-$ 10) with an overall mean of $5.1 \mathrm{~g}$. The range of mid parent heterosis for the trait was from $3.40($ RAH-16 $\times$ DB534) to $77.60($ DHMS $\times$ SNICB75-10) per cent with a mean of 34 .

Out of forty eight crosses, twenty six crosses showed significant positive heterosis. Heterosis over commercial check ranged from -18.40 (RAH-5-10 × RAB4) to 45.90 (DHMS $\times$ SNICB75-10) per cent with a mean of 3.5. Seven and nine hybrids recorded significant positive and negative heterosis over commercial check. Significant positive heterosis over mid parent and commercial check was reported by Mallikarjun (2005), Somashekhar (2006) and Deepakbabu (2007).

Considerable amount of variability was found for plant height in hirsutum lines compared to barbadense lines. The range observed in case of hirsutum lines was from $129.2 \mathrm{~cm}$ (RAH 2510) to $212.2 \mathrm{~cm}$ (DHMS), while it was from $127.7 \mathrm{~cm}$ (RAB 8) to $161.1 \mathrm{~cm}$ (DB534) in barbadense line. The range in observed among hirsutum lines was much wider indicating large variability among the genotypes. The mean was also higher for hirsutums $(169.7 \mathrm{~cm})$ than barbadense lines $(148.8 \mathrm{~cm})$. The variation among the hybrids was from $170.7($ DHMS $\times$ RAB8) to $247.2 \mathrm{~cm}$
(RAH-13-86 $\times$ RAB-4) with an overall mean of $219.7 \mathrm{~cm}$. The heterosis of crosses over mid parent was ranged from $0.4(\mathrm{DHMS} \times \mathrm{RAB} 8)$ to 80.1 (RAH-25-17 $\times$ RAB8) per cent with mean value of 38.9 . Thirty crosses exhibited significant positive heterosis over mid parent. The heterosis of crosses over commercial check varied from -28.9 (DHMS $\times$ RAB8) to $0.3 \mathrm{~cm}$ RAH-13-86 $\times$ RAB-4) with an overall mean of -10.9. Most of the crosses contributed to significant negative heterosis over commercial check. Significant positive heterosis over mid parent was reported by Bhatade et al., (1992) Maisuria et al., (2006), Deosarkar et al., (2009) and Pranesh (2014).

The mean of number of monopodia per plant for hirsutum lines (1.9) was found to be higher than barbadense lines (0.7). High range for this trait in hirsutum lines (1.2 to 2.4) than barbadense lines (0.6 to 0.9). The lowest value was observed in barbadense line SNICB 75-10 and highest value observed in hirsutum line RAH 16. The cross DHMS $\times$ SNICB7510 recorded lower mean value (0.6) and RAH$13-86 \times \mathrm{RAB} 8$ recorded the highest mean value (2.8) with the overall mean for the character was 2.00. Heterosis in negative direction is desirable for monopodia but only one cross exhibited significant negative heterosis over mid parent and the range of heterosis of $-49.5($ DHMS $\times$ SNICB75-10) per cent with a mean of 57.2. With respect to useful heterosis, two crosses manifested significant negative heterosis and the heterosis values ranged from -68.6 (DHMS $\times$ SNICB75-10) to 57.1 (RAH-13-86 $\times$ RAB8) with a mean of 16.20. Negative heterosis over mid parent and commercial check was reported by Shanmugavalli and Vijendradas (1995), Reddy (2001) and Punitha and Ravikesavan (2004). This negative heterosis for number of monopodia is highly desirable because increase in the monopodia makes the plant bushy and robust thus causing a wasteful increase in the space occupied by the plant. 
Table.1 Per se performance of parental lines representing hirsutum and barbadense groups

\begin{tabular}{|c|c|c|c|c|c|c|c|c|c|c|c|c|c|c|c|c|c|c|}
\hline 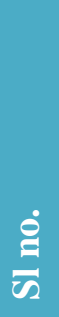 & 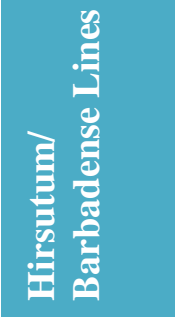 & 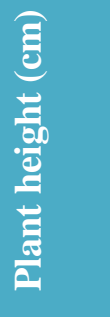 & 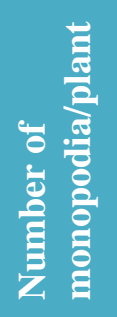 & 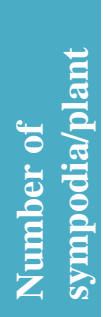 & 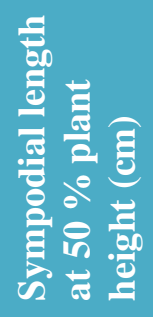 & 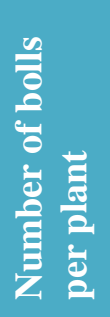 & 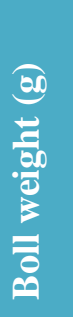 & 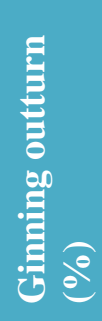 & 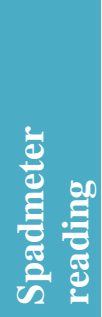 & 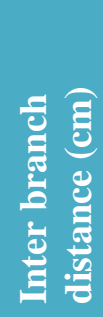 & 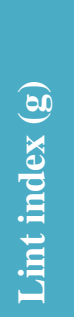 & 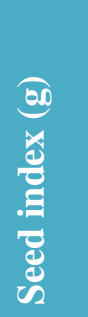 & 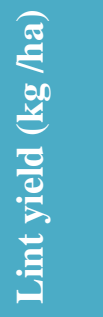 & 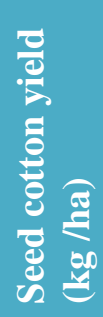 & 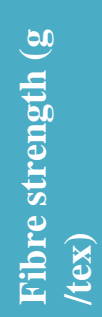 & 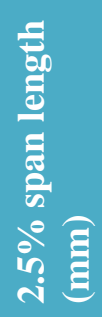 & 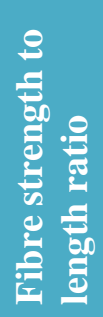 & 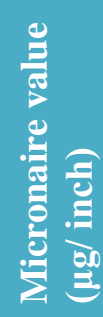 \\
\hline \multicolumn{19}{|c|}{ Hirsutum lines } \\
\hline 1 & DH2752 & 160.9 & 2.4 & 21.0 & 65.0 & 36.0 & 4.4 & 35.4 & 46.0 & 6.5 & 4.9 & 8.8 & 897 & 1554 & 21.3 & 25.9 & 0.8 & 4.5 \\
\hline 2 & DHMS & 212.2 & 1.6 & 23.7 & 49.0 & 34.5 & 4.6 & 37.1 & 43.0 & 8.0 & 5.3 & 9.1 & 913 & 1723 & 21.1 & 26.8 & 0.8 & 4.8 \\
\hline 3 & $\begin{array}{l}\text { RAH-13- } \\
86\end{array}$ & 169.7 & 2.3 & 19.8 & 46.9 & 23.5 & 3.6 & 34.9 & 43.0 & 9.5 & 6.2 & 11.6 & 790 & 1366 & 21.9 & 25.5 & 0.8 & 4.2 \\
\hline 4 & RAH-16 & 173.5 & 2.4 & 19.9 & 47.5 & 35.1 & 4.3 & 34.7 & 42.5 & 7.5 & 4.7 & 8.7 & 1065 & 1733 & 23.1 & 28.3 & 0.8 & 4.4 \\
\hline 5 & $\begin{array}{l}\text { RAH-25- } \\
17\end{array}$ & 129.2 & 1.5 & 18.4 & 45.1 & 26.3 & 4.5 & 36.5 & 46.0 & 8.5 & 5.4 & 9.5 & 843 & 1397 & 21.3 & 29.7 & 0.7 & 4.6 \\
\hline 6 & RAH-370 & 162.2 & 1.2 & 21.5 & 46.6 & 27.3 & 4.2 & 34.3 & 47.5 & 10.5 & 5.5 & 10.5 & 869 & 1410 & 23.0 & 29.4 & 0.8 & 4.5 \\
\hline 7 & $\begin{array}{l}\text { RAH-5- } \\
10\end{array}$ & 197.4 & 1.9 & 24.4 & 49.0 & 20.6 & 3.8 & 31.1 & 44.5 & 6.5 & 4.5 & 9.9 & 701 & 1346 & 22.8 & 28.0 & 0.8 & 4.7 \\
\hline \multirow[t]{4}{*}{8} & DH-37 & 153.0 & 2.4 & 18.8 & 42.4 & 30.1 & 3.8 & 32.1 & 41.5 & 8.5 & 4.8 & 10.1 & 688 & 1248 & 21.0 & 28.4 & 0.8 & 4.7 \\
\hline & Mean & 169.7 & 1.9 & 20.9 & 48.9 & 29.2 & 4.1 & 34.5 & 44.3 & 8.2 & 5.1 & 9.7 & 845 & 1472 & 21.9 & 27.8 & 0.8 & 4.6 \\
\hline & Min & 129.2 & 1.2 & 18.4 & 42.4 & 20.6 & 3.6 & 31.1 & 41.5 & 6.5 & 4.5 & 8.7 & 688 & 1248 & 21.0 & 25.5 & 0.7 & 4.2 \\
\hline & Max & 212.2 & 2.4 & 24.4 & 65.0 & 36.0 & 4.6 & 37.1 & 47.5 & 10.5 & 6.2 & 11.6 & 1065 & 1733 & 23.1 & 29.7 & 0.8 & 4.8 \\
\hline \multicolumn{19}{|c|}{ Barbadense Lines } \\
\hline 1 & DB534 & 161.1 & 0.8 & 21.3 & 42.8 & 30.6 & 3.8 & 33.5 & 44.0 & 10.5 & 5.7 & 11.3 & 608 & 1305 & 28.6 & 33.0 & 0.9 & 3.8 \\
\hline 2 & $\begin{array}{l}\text { SNICB75 } \\
-10\end{array}$ & 159.7 & 0.6 & 23.8 & 53.5 & 30.6 & 3.5 & 32.9 & 49.0 & 11.0 & 5.2 & 10.5 & 574 & 1286 & 28.7 & 36.0 & 0.8 & 3.3 \\
\hline 3 & RAB-4 & 146.9 & 0.9 & 25.2 & 43.5 & 24.5 & 3.4 & 31.9 & 44.0 & 9.5 & 5.5 & 11.8 & 601 & 1263 & 29.2 & 33.1 & 0.9 & 3.5 \\
\hline \multirow[t]{4}{*}{4} & RAB-8 & 127.7 & 0.7 & 23.2 & 41.5 & 25.0 & 3.3 & 32.9 & 44.0 & 7.5 & 4.8 & 9.7 & 709 & 1419 & 29.6 & 31.0 & 1.0 & 3.8 \\
\hline & Mean & 148.8 & 0.7 & 23.4 & 45.3 & 27.7 & 3.5 & 32.8 & 45.3 & 9.6 & 5.3 & 10.8 & 623 & 1318 & 29.0 & 33.3 & 0.9 & 3.6 \\
\hline & Min & 127.7 & 0.6 & 21.3 & 41.5 & 24.5 & 3.3 & 31.9 & 44.0 & 7.5 & 4.8 & 9.7 & 574 & 1263 & 28.6 & 31.0 & 0.8 & 3.3 \\
\hline & Max & 161.1 & 0.9 & 25.2 & 53.5 & 30.6 & 3.8 & 33.5 & 49.0 & 11.0 & 5.7 & 11.8 & 709 & 1419 & 29.6 & 36.0 & 1.0 & 3.8 \\
\hline
\end{tabular}


Table.2a Per se performance and heterosis of crosses between lines representing hirsutum and barbadense groups

\begin{tabular}{|c|c|c|c|c|c|c|c|c|c|c|c|c|c|}
\hline \multirow[t]{2}{*}{ SI. No. } & \multirow[t]{2}{*}{ Crosses } & \multicolumn{3}{|c|}{ Seed cotton yield (kg/ha) } & \multicolumn{3}{|c|}{ Lint yield (kg/ha) } & \multicolumn{3}{|c|}{ Number of bolls per plant } & \multicolumn{3}{|c|}{ Boll weight (g) } \\
\hline & & Mean & Hmp & Hec & Mean & Hmp & Hcc & Mean & Hmp & Hec & Mean & Hmp & Hec \\
\hline 1 & $\mathrm{DH} 2752 \times \mathrm{DB} 534$ & 3260 & $92.6^{*}$ & $95.8^{*}$ & 930 & 23.6 & $70.6^{*}$ & 49.4 & $48.3^{*}$ & $29.2^{*}$ & 6.5 & $60.9^{*}$ & $32.7^{*}$ \\
\hline 2 & DH2752 $\times$ SNICB75-10 & 3152 & $89.4^{*}$ & $89.4^{*}$ & 1073 & $45.8^{*}$ & $96.8^{*}$ & 50.8 & $52.7^{*}$ & $33.0^{*}$ & 5.7 & $45.2^{*}$ & $16.3^{*}$ \\
\hline 3 & DH $2752 \times$ RAB 4 & 2489 & $65.2^{*}$ & $49.5^{*}$ & 769 & 2.6 & $41.1^{*}$ & 35.6 & 17.5 & -6.9 & 4.4 & 13.6 & -10.2 \\
\hline 4 & $\mathrm{DH} 2752 \times \mathrm{RAB} 8$ & 2750 & $53.5^{*}$ & $65.2^{*}$ & 977 & 21.6 & $79.2^{*}$ & 43.7 & $43.3^{*}$ & $14.4^{*}$ & 5.9 & $55.5^{*}$ & $20.4^{*}$ \\
\hline 5 & DHMS $\times$ DB534 & 3236 & $98.0^{*}$ & $94.4^{*}$ & 1157 & $52.1^{*}$ & $112.2^{*}$ & 59.7 & $83.6^{*}$ & $56.3^{*}$ & 6.6 & $58.3^{*}$ & $33.7^{*}$ \\
\hline 6 & DHMS $\times$ SNICB75-10 & 3500 & $113.5^{*}$ & $110.2^{*}$ & 1300 & $74.8^{*}$ & $138.5^{*}$ & 58.6 & $80.2^{*}$ & $53.4^{*}$ & 7.2 & $77.6^{*}$ & $45.9^{*}$ \\
\hline 7 & DHMS $\times$ RAB -4 & 2566 & $61.5^{*}$ & $54.2^{*}$ & 845 & 11.5 & $55.0^{*}$ & 43.3 & $46.8^{*}$ & 13.4 & 5.9 & $48.1^{*}$ & $19.4^{*}$ \\
\hline 8 & DHMS $\times$ RAB 8 & 2996 & $76.6^{*}$ & $80.0^{*}$ & 1068 & $31.7^{*}$ & $96.0^{*}$ & 53.5 & $79.9^{*}$ & $40.1^{*}$ & 5.7 & $46.2^{*}$ & $16.3^{*}$ \\
\hline 9 & RAH-13-86 × DB534 & 2103 & $87.7^{*}$ & 26.3 & 744 & 6.5 & $36.4^{*}$ & 43.5 & $61.1^{*}$ & $13.9^{*}$ & 4.6 & $24.2^{*}$ & -7.1 \\
\hline 10 & RAH-13-86 × SNICB75-10 & 2649 & $79.7^{*}$ & $59.1^{*}$ & 1002 & $47.0^{*}$ & $83.9^{*}$ & 47.5 & $75.9^{*}$ & $24.3^{*}$ & 5.0 & $39.4^{*}$ & 1.0 \\
\hline 11 & RAH-13-86 × RAB-4 & 2648 & $81.4^{*}$ & $59.1^{*}$ & 981 & $41.0^{*}$ & $79.9^{*}$ & 49.2 & $105.2^{*}$ & $28.8^{*}$ & 5.2 & $49.6^{*}$ & 6.1 \\
\hline 12 & RAH-13-86 × RAB8 & 1953 & 25.5 & 17.3 & 658 & -12.2 & 20.7 & 41.2 & $70.1^{*}$ & 7.9 & 4.9 & $41.6^{*}$ & -1.0 \\
\hline 13 & RAH-16 × DB534 & 1858 & 16.8 & 11.6 & 719 & -14.0 & 31.9 & 38.4 & 17.1 & 0.5 & 4.2 & 3.4 & -15.3 \\
\hline 14 & RAH-16 $\times$ SNICB75-10 & 2737 & $72.0^{*}$ & $64.4^{*}$ & 993 & 21.2 & $82.2^{*}$ & 37.2 & 13.4 & -2.6 & 5.1 & $30.3^{*}$ & 3.1 \\
\hline 15 & RAH-16 × RAB-4 & 2411 & $69.8^{*}$ & $44.8^{*}$ & 825 & -1.0 & $51.4^{*}$ & 39.8 & $33.7^{*}$ & 4.2 & 5.1 & $32.9^{*}$ & 3.1 \\
\hline 16 & RAH-16 $\times$ RAB 8 & 1814 & 7.3 & 9.0 & 726 & -18.1 & $33.2^{*}$ & 38.8 & $29.2^{*}$ & 1.6 & 4.3 & 13.3 & -13.3 \\
\hline 17 & RAH-25-17 × DB534 & 3178 & $122.9^{*}$ & $90.9^{*}$ & 1080 & $48.9^{*}$ & $98.2^{*}$ & 52.1 & $83.4^{*}$ & $36.4^{*}$ & 6.4 & $55.4^{*}$ & $29.6^{*}$ \\
\hline 18 & RAH-25-17 × SNICB75-10 & 1889 & 42.2 & 13.5 & 712 & 0.5 & $30.6^{*}$ & 41.0 & $44.3^{*}$ & 7.2 & 4.5 & 12.8 & $-9.2^{*}$ \\
\hline 19 & RAH-25-17 × RAB4 & 1828 & 35.3 & 9.8 & 754 & 4.4 & $38.3^{*}$ & 35.9 & $41.5^{*}$ & -6.0 & 4.5 & 13.5 & $-9.2^{*}$ \\
\hline 20 & RAH-25-17 × RAB8 & 2369 & $50.4^{*}$ & $42.3^{*}$ & 737 & -5.0 & $35.2^{*}$ & 50.5 & $96.9^{*}$ & $32.1^{*}$ & 4.8 & $24.4^{*}$ & -2.0 \\
\hline 21 & RAH-370 × DB534 & 2095 & $50.6^{*}$ & 25.9 & 785 & 6.2 & $43.9^{*}$ & 43.2 & $49.5^{*}$ & 13.1 & 4.4 & 10.5 & -11.2 \\
\hline 22 & RAH-370 $\times$ SNICB75-10 & 2824 & $99.4^{*}$ & $69.6^{*}$ & 872 & 20.7 & $59.9^{*}$ & 40.1 & $38.8^{*}$ & 5.0 & 4.5 & $16.7^{*}$ & $-9.2^{*}$ \\
\hline 23 & RAH-370 $\times$ RAB-4 & 2801 & $103.6^{*}$ & $68.3^{*}$ & 948 & 29.0 & $73.9^{*}$ & 42.6 & $64.6^{*}$ & 11.5 & 4.9 & $29.3^{*}$ & -1.0 \\
\hline 24 & RAH-370 $\times$ RAB- 8 & 2265 & $54.1^{*}$ & $36.1^{*}$ & 766 & -2.9 & $40.6^{*}$ & 48.4 & $85.3^{*}$ & $26.7^{*}$ & 4.9 & $32.4^{*}$ & 0.0 \\
\hline 25 & RAH-5-10 × DB534 & 2401 & $68.6^{*}$ & $44.2^{*}$ & 854 & 30.5 & $56.7^{*}$ & 44.5 & $73.8^{*}$ & $16.4^{*}$ & 5.0 & $33.0^{*}$ & 1.0 \\
\hline 26 & RAH-5-10 $\times$ SNICB75-10 & 1674 & 18.9 & 0.6 & 580 & -9.0 & 6.4 & 35.0 & $36.9^{*}$ & -8.4 & 4.3 & $18.9^{*}$ & -12.2 \\
\hline 27 & RAH-5-10 $\times$ RAB4 & 1783 & 23.3 & 7.1 & 593 & -8.8 & 8.8 & 37.3 & $65.4^{*}$ & -2.4 & 4.0 & 11.7 & -18.4 \\
\hline 28 & RAH-5-10 $\times$ RAB- 8 & 2368 & $58.1^{*}$ & $42.2^{*}$ & 800 & 13.6 & $46.8^{*}$ & 43.6 & $91.0^{*}$ & $14.0^{*}$ & 5.1 & $44.9^{*}$ & 3.1 \\
\hline 29 & DH-37 × DB534 & 2677 & $86.8^{*}$ & $60.8^{*}$ & 919 & $41.8^{*}$ & $68.5^{*}$ & 49.5 & $63.0^{*}$ & $29.5^{*}$ & 5.5 & $47.2^{*}$ & $12.2^{*}$ \\
\hline 30 & DH-37 $\times$ SNICB75-10 & 1758 & 30.2 & 5.6 & 562 & -11.0 & 3.0 & 37.3 & $22.8^{*}$ & -2.5 & 4.2 & 14.8 & -15.3 \\
\hline 31 & DH-37 × RAB4 & 1913 & $51.2^{*}$ & 14.9 & 597 & -7.4 & 9.4 & 38.3 & $40.3^{*}$ & 0.3 & 4.8 & $33.1^{*}$ & -3.1 \\
\hline \multirow[t]{6}{*}{32} & DH-37 $\times$ RAB- 8 & 2788 & $89.8^{*}$ & $67.5^{*}$ & 948 & $35.8^{*}$ & $73.9^{*}$ & 45.8 & $66.1^{*}$ & $19.8^{*}$ & 5.2 & $48.7^{*}$ & 6.1 \\
\hline & Mean & 2460 & 64.9 & 47.8 & 852 & 16.3 & 56.4 & 44.2 & 56.9 & 15.7 & 5.1 & 34.0 & 3.5 \\
\hline & Min & 1674 & 7.3 & 0.6 & 562 & -18.1 & 3.0 & 35.0 & 13.4 & -8.4 & 4.0 & 3.4 & -18.4 \\
\hline & Max & 3500 & 122.9 & 110.2 & 1300 & 74.8 & 138.5 & 59.7 & 105.2 & 56.3 & 7.2 & 77.6 & 45.9 \\
\hline & SEd & 367.54 & 318.3 & 367.54 & 117 & 101.32 & 117 & 3.61 & 3.13 & 3.61 & 0.34 & 0.29 & 0.34 \\
\hline & CD@5\% & 741.22 & 641.91 & 741.22 & 235.95 & 204.34 & 235.95 & 7.28 & 6.31 & 7.28 & 0.68 & 0.59 & 0.68 \\
\hline
\end{tabular}


Table.2b Per se performance and heterosis of crosses between lines representing hirsutum and barbadense groups

\begin{tabular}{|c|c|c|c|c|c|c|c|c|c|c|c|c|c|}
\hline \multirow[t]{2}{*}{ SI no. } & \multirow[t]{2}{*}{ Crosses } & \multicolumn{3}{|c|}{ Plant height (cm) } & \multicolumn{3}{|c|}{ Number of monopodia } & \multicolumn{3}{|c|}{ Number of sympodia } & \multicolumn{3}{|c|}{$\begin{array}{c}\text { Sympodial length at } 50 \% \\
\text { plant height }(\mathrm{cm})\end{array}$} \\
\hline & & Mean & Hmp & Hec & Mean & Hmp & Hec & Mean & Hmp & Hec & Mean & Hmp & Hec \\
\hline 1 & DH2752 $\times$ DB534 & 216.4 & $34.5^{*}$ & $-9.8^{*}$ & 1.9 & 15.5 & 5.7 & 35.7 & $68.8^{*}$ & 6.1 & 56.2 & 4.2 & $22.1^{*}$ \\
\hline 2 & DH $2752 \times$ SNICB75-10 & 233.5 & $47.3^{*}$ & -2.7 & 1.5 & -1.0 & -14.3 & 33.4 & $49.0^{*}$ & -0.7 & 71.3 & $20.3^{*}$ & $54.9^{*}$ \\
\hline 3 & DH $2752 \times$ RAB 4 & 224.7 & $45.8^{*}$ & $-6.4^{*}$ & 1.9 & 15.8 & 8.6 & 32.5 & $41.1^{*}$ & -3.3 & 62.4 & $15.1^{*}$ & $35.7^{*}$ \\
\hline 4 & DH2752 $\times$ RAB 8 & 196.9 & $36.6^{*}$ & $-18.0^{*}$ & 2.1 & $36.1^{*}$ & $20.0^{*}$ & 30.7 & $39.2^{*}$ & -8.6 & 76.3 & $43.2^{*}$ & $65.8^{*}$ \\
\hline 5 & DHMS $\times$ DB534 & 230.1 & $23.3^{*}$ & -4.1 & 2.5 & $112.9^{*}$ & $42.9^{*}$ & 38.0 & $69.1^{*}$ & $13.1^{*}$ & 72.9 & $58.9^{*}$ & $58.5^{*}$ \\
\hline 6 & DHMS $\times$ SNICB75-10 & 215.4 & $18.2^{*}$ & $-10.3^{*}$ & 0.6 & $-49.5^{*}$ & $-68.6^{*}$ & 43.0 & $81.2^{*}$ & $28.0^{*}$ & 81.6 & $59.2^{*}$ & $77.4^{*}$ \\
\hline 7 & DHMS $\times$ RAB -4 & 213.4 & $18.9^{*}$ & $-11.1^{*}$ & 2.5 & $105.8^{*}$ & $42.9^{*}$ & 33.5 & $37.4^{*}$ & -0.3 & 64.2 & $38.7^{*}$ & $39.5^{*}$ \\
\hline 8 & DHMS $\times$ RAB 8 & 170.7 & 0.4 & $-28.9^{*}$ & 2.4 & $111.1^{*}$ & $37.1^{*}$ & 37.0 & $58.1^{*}$ & $10.1^{*}$ & 66.6 & $47.1^{*}$ & $44.8^{*}$ \\
\hline 9 & RAH-13-86 × DB534 & 210.5 & $27.4^{*}$ & $-12.3^{*}$ & 1.5 & -4.2 & -17.1 & 37.4 & $81.7^{*}$ & $11.2^{*}$ & 71.8 & $59.9^{*}$ & $56.0^{*}$ \\
\hline 10 & RAH-13-86 × SNICB75-10 & 227.0 & $40.7^{*}$ & -5.4 & 2.2 & $51.9^{*}$ & $22.9^{*}$ & 40.1 & $83.9^{*}$ & $19.3^{*}$ & 63.7 & $26.7^{*}$ & $38.4^{*}$ \\
\hline 11 & RAH-13-86 × RAB-4 & 247.2 & $56.1^{*}$ & 3.0 & 2.4 & $54.2^{*}$ & $37.1^{*}$ & 37.5 & $66.9^{*}$ & $11.6^{*}$ & 74.7 & $65.0^{*}$ & $62.3^{*}$ \\
\hline 12 & RAH-13-86 × RAB8 & 229.2 & $54.2^{*}$ & -4.5 & 2.8 & $87.9^{*}$ & $57.1^{*}$ & 31.8 & $47.8^{*}$ & -5.5 & 73.4 & $65.9^{*}$ & $59.6^{*}$ \\
\hline 13 & RAH-16 × DB534 & 228.3 & $35.4^{*}$ & -4.9 & 2.6 & $64.2^{*}$ & $45.7^{*}$ & 28.8 & $39.6^{*}$ & $-14.4^{*}$ & 61.0 & $35.2^{*}$ & $32.6^{*}$ \\
\hline 14 & RAH-16 $\times$ SNICB75-10 & 172.7 & 2.7 & $-28.1^{*}$ & 1.9 & 29.7 & 8.6 & 24.9 & 14.0 & $-25.9^{*}$ & 62.0 & $22.8^{*}$ & $34.8^{*}$ \\
\hline 15 & RAH- $16 \times$ RAB- 4 & 204.4 & $26.4^{*}$ & $-14.9^{*}$ & 2.0 & 24.2 & 14.3 & 26.9 & $19.2^{*}$ & $-20.1^{*}$ & 72.3 & $58.8^{*}$ & $57.1^{*}$ \\
\hline 16 & RAH-16 × RAB8 & 225.8 & $48.7^{*}$ & $-5.9^{*}$ & 2.1 & $40.0^{*}$ & $20.0^{*}$ & 31.6 & $46.7^{*}$ & -6.0 & 62.4 & $40.1^{*}$ & $35.5^{*}$ \\
\hline 17 & RAH-25-17 $\times$ DB534 & 232.2 & $61.7^{*}$ & -3.3 & 2.4 & $120.7^{*}$ & $37.1^{*}$ & 37.2 & $87.2^{*}$ & $10.6^{*}$ & 68.3 & $55.6^{*}$ & $48.4^{*}$ \\
\hline 18 & RAH-25-17 × SNICB75-10 & 233.8 & $66.7^{*}$ & -2.6 & 2.3 & $127.1^{*}$ & $31.4^{*}$ & 34.1 & $61.7^{*}$ & 1.5 & 70.9 & $43.9^{*}$ & $54.1^{*}$ \\
\hline 19 & RAH-25-17 $\times$ RAB4 & 210.9 & $52.9^{*}$ & $-12.1^{*}$ & 1.9 & $64.5^{*}$ & 8.6 & 31.4 & $44.3^{*}$ & -6.5 & 76.1 & $71.9^{*}$ & $65.4^{*}$ \\
\hline 20 & RAH-25-17 $\times$ RAB8 & 231.0 & $80.1^{*}$ & -3.8 & 2.5 & $131.0^{*}$ & $40.0^{*}$ & 29.6 & $42.4^{*}$ & $-11.9^{*}$ & 62.9 & $45.3^{*}$ & $36.6^{*}$ \\
\hline 21 & RAH-370 × DB534 & 223.1 & $36.4^{*}$ & $-7.0^{*}$ & 1.7 & $68.8^{*}$ & -5.7 & 36.0 & $68.2^{*}$ & 7.1 & 72.9 & $63.2^{*}$ & $58.4^{*}$ \\
\hline 22 & RAH-370 $\times$ SNICB75-10 & 223.9 & $37.6^{*}$ & $-6.7^{*}$ & 1.7 & $88.2^{*}$ & -2.9 & 31.2 & $37.6^{*}$ & -7.3 & 66.9 & $33.8^{*}$ & $45.4^{*}$ \\
\hline 23 & RAH-370 $\times$ RAB- 4 & 205.4 & $31.3^{*}$ & $-14.4^{*}$ & 1.4 & 35.5 & $-20.0^{*}$ & 27.9 & $19.6^{*}$ & $-17.0^{*}$ & 67.3 & $49.4^{*}$ & $46.2^{*}$ \\
\hline 24 & RAH-370 × RAB- 8 & 226.6 & $54.3^{*}$ & $-5.6^{*}$ & 2.4 & $151.4^{*}$ & $34.3^{*}$ & 26.2 & $17.4^{*}$ & $-22.0^{*}$ & 72.4 & $64.4^{*}$ & $57.3^{*}$ \\
\hline 25 & RAH-5-10 × DB534 & 231.8 & $25.9^{*}$ & -3.4 & 1.9 & $39.0^{*}$ & 5.7 & 33.5 & $46.6^{*}$ & -0.3 & 66.6 & $45.1^{*}$ & $44.8^{*}$ \\
\hline 26 & RAH-5-10 $\times$ SNICB75-10 & 232.5 & $28.3^{*}$ & -3.1 & 2.3 & $85.5^{*}$ & $31.4^{*}$ & 28.1 & $16.6^{*}$ & $-16.4^{*}$ & 77.6 & $51.4^{*}$ & $68.7^{*}$ \\
\hline 27 & RAH-5-10 × RAB4 & 225.5 & $27.2^{*}$ & $-6.0^{*}$ & 2.4 & $72.2^{*}$ & $34.3^{*}$ & 29.1 & $17.4^{*}$ & $-13.4^{*}$ & 66.4 & $43.6^{*}$ & $44.3^{*}$ \\
\hline 28 & RAH-5-10 × RAB-8 & 218.2 & $32.2^{*}$ & $-9.1^{*}$ & 1.7 & 27.5 & -5.7 & 36.1 & $51.8^{*}$ & 7.4 & 68.6 & $51.6^{*}$ & $49.1^{*}$ \\
\hline 29 & DH-37 × DB534 & 223.2 & $42.0^{*}$ & $-7.0^{*}$ & 1.7 & 4.0 & -5.7 & 37.3 & $85.8^{*}$ & $10.9^{*}$ & 56.6 & $32.8^{*}$ & $23.0^{*}$ \\
\hline 30 & DH-37 × SNICB75-10 & 235.0 & $50.4^{*}$ & -2.1 & 2.6 & $71.1^{*}$ & $45.7^{*}$ & 26.0 & $22.1^{*}$ & $-22.6^{*}$ & 57.0 & $18.8^{*}$ & $23.9^{*}$ \\
\hline 31 & $\mathrm{DH}-37 \times \mathrm{RAB} 4$ & 222.5 & $48.3^{*}$ & $-7.3^{*}$ & 2.2 & $34.7^{*}$ & $25.7^{*}$ & 30.7 & $39.5^{*}$ & $-8.8^{*}$ & 58.8 & $36.6^{*}$ & $27.7^{*}$ \\
\hline \multirow[t]{6}{*}{32} & DH-37 $\times$ RAB- 8 & 210.9 & $52.6^{*}$ & $-12.1^{*}$ & 1.8 & 14.8 & 0.0 & 35.7 & $70.0^{*}$ & 6.1 & 67.1 & $59.7^{*}$ & $45.9^{*}$ \\
\hline & Mean & 219.7 & 38.9 & 5.1 & 2.0 & 57.2 & 16.2 & 32.9 & 49.1 & -2.1 & 67.8 & 44.6 & 47.3 \\
\hline & Min & 170.7 & 0.4 & -18.3 & 0.6 & -49.5 & -68.6 & 24.9 & 14.0 & -25.9 & 56.2 & 4.2 & 22.1 \\
\hline & $\operatorname{Max}$ & 247.2 & 80.1 & 18.3 & 2.8 & 151.4 & 57.1 & 43.0 & 87.2 & 28.0 & 81.6 & 71.9 & 77.4 \\
\hline & SEd & 6.62 & 5.73 & 6.62 & 0.26 & 0.23 & 0.26 & 2.09 & 1.81 & 2.09 & 2.66 & 2.31 & 2.66 \\
\hline & CD@5\% & 13.35 & 11.57 & 13.35 & 0.53 & 0.46 & 0.53 & 4.21 & 3.65 & 4.21 & 5.37 & 4.65 & 5.37 \\
\hline
\end{tabular}


Table.2c Per se performance and heterosis of crosses between lines representing hirsutum and barbadense groups

\begin{tabular}{|c|c|c|c|c|c|c|c|c|c|c|c|c|c|c|c|c|}
\hline \multirow[t]{2}{*}{$\begin{array}{c}\text { SI } \\
\text { no. }\end{array}$} & \multirow[t]{2}{*}{ Crosses } & \multicolumn{3}{|c|}{$\begin{array}{l}\text { Inter branch distance } \\
(\mathbf{c m})\end{array}$} & \multicolumn{3}{|c|}{ Ginning outturn (\%) } & \multicolumn{3}{|c|}{ SPAD meter reading } & \multicolumn{3}{|c|}{ Lint index (g) } & \multicolumn{3}{|c|}{ Seed index (g) } \\
\hline & & Mean & Hmp & Hec & Mean & Hmp & Hec & Mean & Hmp & Hec & Mean & Hmp & Hec & Mean & Hmp & Hec \\
\hline 1 & DH2752 × DB534 & 8.1 & -5.3 & -11.0 & 30.7 & -10.9 & -6.1 & 42.9 & $-4.7^{*}$ & $-5.0^{*}$ & 5.6 & $3.7^{*}$ & -0.9 & 12.5 & $23.4^{*}$ & 4.2 \\
\hline 2 & DH2752 $\times$ SNICB75-10 & 8.5 & -2.9 & -6.1 & 34.0 & -0.5 & 4.1 & 46.2 & -2.9 & 2.3 & 6.3 & $24.8^{*}$ & 12.5 & 12.3 & $26.2^{*}$ & 2.5 \\
\hline 3 & DH $2752 \times$ RAB 4 & 9.8 & $22.5^{*}$ & 8.3 & 32.8 & -2.6 & 0.3 & 42.0 & -6.7 & -6.9 & 6.3 & 20.6 & 12.5 & 13.0 & $25.3^{*}$ & 8.3 \\
\hline 4 & DH2752 $\times$ RAB 8 & 10.4 & $48.6^{*}$ & $14.9^{*}$ & 35.0 & $2.6^{*}$ & $7.2^{*}$ & 42.0 & -6.7 & $-6.9^{*}$ & 6.7 & $38.6^{*}$ & $19.6^{*}$ & 12.5 & $34.0^{*}$ & 4.2 \\
\hline 5 & DHMS $\times$ DB534 & 8.3 & $-10.3^{*}$ & -8.3 & 37.3 & 5.7 & 14.2 & 43.1 & -1.1 & $-4.5^{*}$ & 8.8 & $65.7^{*}$ & $57.1^{*}$ & 14.8 & $46.2^{*}$ & $23.3^{*}$ \\
\hline 6 & DHMS $\times$ SNICB75-10 & 9.6 & 1.1 & 6.1 & 40.9 & 16.8 & 25.3 & 52.6 & $14.3^{*}$ & $16.6^{*}$ & 9.2 & $81.0^{*}$ & $64.3^{*}$ & 13.3 & $36.4^{*}$ & 10.8 \\
\hline 7 & DHMS $\times$ RAB -4 & 7.7 & $-12.0^{*}$ & $-14.9^{*}$ & 31.7 & -8.3 & $-3.1^{*}$ & 40.0 & $-8.2^{*}$ & -11.4 & 5.7 & 8.0 & 0.9 & 12.3 & 18.1 & 2.1 \\
\hline 8 & DHMS $\times$ RAB 8 & 9.7 & $24.5^{*}$ & $6.6^{*}$ & 35.7 & $1.9^{*}$ & 9.2 & 46.4 & 6.5 & $2.8^{*}$ & 7.6 & $55.6^{*}$ & $35.7^{*}$ & 13.7 & $46.4^{*}$ & 13.8 \\
\hline 9 & RAH-13-86 × DB534 & 10.0 & 0.0 & $10.5^{*}$ & 33.7 & -1.4 & 3.2 & 45.3 & 4.0 & 0.3 & 6.5 & 10.4 & 15.2 & 12.5 & 9.9 & 4.2 \\
\hline 10 & RAH-13-86 × SNICB75-10 & 9.0 & $-12.7^{*}$ & -1.1 & 34.7 & $2.4^{*}$ & 6.3 & 42.0 & -8.7 & -6.9 & 7.2 & $28.9^{*}$ & $28.6^{*}$ & 13.5 & $22.7^{*}$ & 12.5 \\
\hline 11 & RAH-13-86 × RAB-4 & 8.6 & -10.0 & -5.5 & 41.6 & 24.6 & 27.4 & 52.3 & $20.1^{*}$ & $15.9^{*}$ & 9.5 & $65.3^{*}$ & $68.8^{*}$ & 13.3 & 14.4 & 10.8 \\
\hline 12 & RAH-13-86 × RAB8 & 10.3 & $20.6^{*}$ & $13.3^{*}$ & 31.2 & $-8.0^{*}$ & $-4.6^{*}$ & 40.6 & $-6.8^{*}$ & -10.1 & 6.1 & 14.0 & 8.9 & 13.5 & $27.7^{*}$ & 12.5 \\
\hline 13 & RAH-16 × DB534 & 9.2 & 2.2 & 1.7 & 34.5 & 1.0 & 5.5 & 41.0 & -5.2 & $-9.1^{*}$ & 7.4 & $44.6^{*}$ & $31.3^{*}$ & 14.0 & $41.8^{*}$ & $16.7^{*}$ \\
\hline 14 & RAH-16 $\times$ SNICB75-10 & 8.8 & -4.9 & -2.8 & 37.3 & 10.2 & 14.1 & 43.3 & $-5.4^{*}$ & $-4.0^{*}$ & 7.3 & $50.7^{*}$ & $30.4^{*}$ & 12.3 & $28.9^{*}$ & 2.1 \\
\hline 15 & RAH-16 × RAB-4 & 9.1 & 6.5 & 0.0 & 33.8 & 1.4 & 3.4 & 43.1 & -0.3 & -4.4 & 7.4 & $47.4^{*}$ & $31.3^{*}$ & 14.4 & $42.2^{*}$ & 20.0 \\
\hline 16 & RAH-16 × RAB8 & 8.2 & 8.7 & -9.9 & 34.3 & 1.4 & 4.9 & 44.3 & 2.3 & $-1.9^{*}$ & 6.4 & $38.4^{*}$ & $13.4^{*}$ & 12.3 & $35.0^{*}$ & 2.1 \\
\hline 17 & RAH-25-17 × DB534 & 8.3 & $-13.2^{*}$ & $-8.8^{*}$ & 34.5 & $-1.4^{*}$ & 5.5 & 40.4 & -10.3 & $-10.5^{*}$ & 7.1 & $26.6^{*}$ & $26.8^{*}$ & 13.5 & $30.1^{*}$ & 12.5 \\
\hline 18 & RAH-25-17 × SNICB75-10 & 9.3 & -5.1 & 2.2 & 30.7 & -11.6 & -6.1 & 41.0 & $-13.7^{*}$ & $-9.1^{*}$ & 5.7 & 6.1 & 0.9 & 12.8 & $27.6^{*}$ & 6.3 \\
\hline 19 & RAH-25-17 $\times$ RAB4 & 9.6 & 6.1 & 5.5 & 31.0 & -9.2 & -5.1 & 43.5 & $-3.4^{*}$ & $-3.7^{*}$ & 5.9 & 6.7 & 4.5 & 13.1 & $22.8^{*}$ & 8.8 \\
\hline 20 & RAH-25-17 × RAB8 & 7.9 & -1.9 & -13.3 & 32.2 & $-7.2^{*}$ & -1.5 & 44.5 & $-1.1^{*}$ & -1.3 & 6.2 & 20.5 & 9.8 & 13.0 & $35.8^{*}$ & 8.3 \\
\hline 21 & RAH-370 × DB534 & 8.8 & $-16.7^{*}$ & $-3.3^{*}$ & 34.8 & 2.6 & 6.4 & 44.6 & -2.6 & $-1.2^{*}$ & 6.8 & 22.0 & $21.4^{*}$ & 12.8 & $17.2^{*}$ & 6.3 \\
\hline 22 & RAH-370 $\times$ SNICB75-10 & 8.5 & $-20.9^{*}$ & $-6.1^{*}$ & 32.9 & $-2.1^{*}$ & 0.8 & 42.0 & -13.1 & -7.0 & 6.3 & 16.9 & 11.6 & 12.7 & $21.0^{*}$ & 5.8 \\
\hline 23 & RAH-370 × RAB-4 & 9.9 & -1.5 & 8.8 & 32.9 & -0.5 & 0.8 & 42.2 & -7.7 & -6.4 & 6.4 & 16.2 & 13.4 & 13.0 & 16.9 & 8.3 \\
\hline 24 & RAH-370 $\times$ RAB-8 & 9.7 & 7.2 & 6.6 & 32.2 & -4.0 & -1.4 & 45.0 & -1.7 & -0.3 & 6.0 & 16.9 & 7.1 & 12.6 & $25.1^{*}$ & 5.0 \\
\hline 25 & RAH-5-10 × DB534 & 7.8 & -8.2 & -13.8 & 35.6 & 10.1 & $8.9^{*}$ & 45.0 & $1.6^{*}$ & $-0.2^{*}$ & 6.9 & $42.3^{*}$ & $22.3^{*}$ & 12.5 & 17.6 & 4.2 \\
\hline 26 & RAH-5-10 × SNICB75-10 & 9.4 & 7.4 & 3.9 & 31.4 & -2.1 & -4.0 & 40.4 & -13.6 & -10.4 & 6.9 & $49.5^{*}$ & $22.3^{*}$ & 15.0 & $46.3^{*}$ & $25.0^{*}$ \\
\hline 27 & RAH-5-10 $\times$ RAB4 & 9.1 & 13.8 & 0.6 & 32.7 & 3.7 & 0.0 & 42.2 & -4.7 & -6.5 & 5.9 & 25.3 & 5.4 & 12.3 & $12.6^{*}$ & 2.1 \\
\hline 28 & RAH-5-10 × RAB-8 & 8.2 & 16.4 & -9.9 & 33.8 & 5.5 & 3.4 & 45.5 & 2.7 & 0.8 & 6.4 & $45.4^{*}$ & 13.4 & 12.5 & $27.2^{*}$ & 4.2 \\
\hline 29 & DH-37 × DB534 & 8.3 & $-13.2^{*}$ & $-8.8^{*}$ & 34.4 & $4.7^{*}$ & 5.2 & 44.5 & 3.9 & -1.4 & 6.7 & $25.2^{*}$ & 19.6 & 12.8 & 20.0 & 6.3 \\
\hline 30 & DH-37 × SNICB75-10 & 8.8 & -10.3 & -3.3 & 31.7 & -2.4 & -2.9 & 43.9 & -3.1 & -2.8 & 7.4 & $45.3^{*}$ & $31.3^{*}$ & 15.8 & $53.7^{*}$ & $31.3^{*}$ \\
\hline 31 & DH-37 × RAB4 & 9.1 & 0.6 & 0.0 & 32.4 & 1.3 & -0.8 & 43.5 & 1.8 & -3.5 & 6.3 & 19.8 & 12.5 & 13.1 & $20.0^{*}$ & 8.8 \\
\hline \multirow[t]{6}{*}{32} & DH-37 $\times$ RAB- 8 & 8.6 & 6.9 & -5.5 & 32.9 & 1.4 & 0.8 & 44.0 & 3.0 & -2.4 & 6.7 & $38.1^{*}$ & $19.6^{*}$ & 13.7 & $38.9^{*}$ & 13.8 \\
\hline & Mean & 8.9 & 1.4 & -1.4 & 33.9 & 0.8 & 3.8 & 43.7 & -2.2 & 4.0 & 6.8 & 31.9 & 21.0 & 13.2 & 28.5 & 9.6 \\
\hline & Min & 7.7 & -20.9 & -14.9 & 30.7 & -11.6 & -6.1 & 40.0 & -13.7 & -4.9 & 5.6 & 3.7 & -0.9 & 12.3 & 9.9 & 2.1 \\
\hline & Max & 10.4 & 48.6 & 14.9 & 41.6 & 24.6 & 27.4 & 52.6 & 20.1 & 25.2 & 9.5 & 81.0 & 68.8 & 15.8 & 53.7 & 31.3 \\
\hline & SEd & 1.22 & 1.05 & 1.22 & 2.7 & 2.34 & 2.7 & 0.76 & 0.66 & 0.76 & 0.77 & 0.67 & 0.77 & 1.26 & 1.09 & 1.26 \\
\hline & CD@5\% & 2.46 & 2.13 & 2.46 & 5.44 & 4.71 & 5.44 & 1.54 & 1.33 & 1.54 & 1.55 & 1.35 & 1.55 & 2.54 & 2.2 & 2.54 \\
\hline
\end{tabular}


Table.3 Determining potentiality of hirsutum vs barbadense heterotic boxes (HB) based on predicted double cross performance

\begin{tabular}{|c|c|c|}
\hline HB I & \multicolumn{2}{|c|}{$($ DHMS $\times$ SNICB75-10) $x($ DH2752 $\times$ DB534) } \\
\hline & Single crosses & Seed cotton yield (kg/ha) \\
\hline 1 & DHMS $\times$ SNICB75-10 & 3500 \\
\hline 2 & DHMS $\times$ DB534 & 3236 \\
\hline 3 & DH2752 $\times$ DB534 & 3260 \\
\hline \multirow[t]{2}{*}{4} & DH $2752 \times$ SNICB75-10 & 3152 \\
\hline & Predicted double cross mean & 3287 \\
\hline HB II & \multicolumn{2}{|c|}{$($ RAH-13-86 $\times$ SNICB75-10 $) \times($ DHMS $\times$ RAB-4 $)$} \\
\hline & Single crosses & Seed cotton yield ( $\mathrm{kg} / \mathrm{ha})$ \\
\hline 1 & RAH-13-86 × SNICB75-10 & 2649 \\
\hline 2 & RAH-13-86 × RAB-4 & 2648 \\
\hline 3 & DHMS $\times$ SNICB75-10 & 3500 \\
\hline \multirow[t]{2}{*}{4} & DHMS $\times$ RAB -4 & 2566 \\
\hline & Predicted double cross mean & 2841 \\
\hline HB III & \multicolumn{2}{|c|}{$($ RAH-25-17 $\times$ RAB-8 $) \times($ DH-37 $\times$ DB534 $)$} \\
\hline & Single crosses & Seed cotton yield $(\mathrm{kg} / \mathrm{ha})$ \\
\hline 1 & RAH-25-17 $\times$ RAB8 & 2369 \\
\hline 2 & RAH-25-17 × DB534 & 3178 \\
\hline 3 & DH-37 $\times$ RAB- 8 & 2788 \\
\hline \multirow[t]{2}{*}{4} & DH-37 × DB534 & 2677 \\
\hline & Predicted double cross mean & 2753 \\
\hline HB IV & \multicolumn{2}{|c|}{$($ RAH-16 $\times$ RAB-4 $) \times($ RAH-370 $\times$ SNICB75-10 $)$} \\
\hline & Single crosses & Seed cotton yield ( $\mathrm{kg} / \mathrm{ha})$ \\
\hline 1 & RAH-16 $\times$ RAB-4 & 2411 \\
\hline 2 & RAH-16 $\times$ SNICB75-10 & 2737 \\
\hline 3 & RAH-370 × RAB-4 & 2801 \\
\hline \multirow[t]{2}{*}{4} & RAH-370 $\times$ SNICB75-10 & 2824 \\
\hline & Predicted double cross mean & 2693 \\
\hline HB V & \multicolumn{2}{|c|}{$($ RAH-510 $\times$ RAB-4) $X($ RAH-370 $\times$ DB534 $)$} \\
\hline & Single crosses & Seed cotton yield $(\mathrm{kg} / \mathrm{ha})$ \\
\hline 1 & RAH-5-10 × RAB-4 & 1783 \\
\hline 2 & RAH-5-10 × DB534 & 2401 \\
\hline 3 & RAH-370 × RAB-4 & 2801 \\
\hline 4 & RAH-370 × DB534 & 2095 \\
\hline & Predicted double cross mean & 2270 \\
\hline
\end{tabular}

The range for number of sympodia per plant was 18.4 to 24.4 and 21.3 to 25.2 in hirsutum and barbadense lines, respectively. The lowest value was observed in hirsutum line RAH 25-17 and highest value observed in barbadense line RAB 4. The mean observed among hirsutum lines (20.9) was much wider compared to barbadense lines (23.4). The range of this character among the crosses was from $24.9(\mathrm{RAH}-16 \times \mathrm{SNICB} 75-10)$ to 43.0 
$($ DHMS $\times$ SNICB75-10) with an overall mean of 32.9. The expression of heterosis over mid parent values in hybrids ranged from 14.0 $(\mathrm{RAH}-16 \times$ SNICB75-10) to $87.2(\mathrm{RAH}-25-$ $17 \times$ DB534) per cent with a mean of 49.1. Thirty one crosses exhibited significant positive heterosis. With respect to standard heterosis, seventeen crosses depicted significant heterosis with a minimum of -25.9 and maximum of 28 per cent was recorded by the crosses RAH-16 $\times$ SNICB75-10 and DHMS $\times$ SNICB75-10, respectively. The mean heterosis was -2.1. Significant positive heterosis over mid parent was reported by Reddy (2001), Punitha and Ravikesavan (2004). Significant positive heterosis over commercial check was reported by Neelima (2002), Maisuria et al., (2006) and Saifullah et al., (2014).

The range observed for sympodial length at 50 per cent plant height was higher in hirsutum lines $(42.4$ to $65.0 \mathrm{~cm})$ than the barbadense lines (41.5 to $53.5 \mathrm{~cm}$ ). The mean for this trait was also higher in hirsutum lines $(48.9 \mathrm{~cm})$ than barbadense $(45.3 \mathrm{~cm})$. The lowest and highest reproductive points on sympodia was observed in barbadense line RAB 8 and hirsutum line DH2752 respectively. The mean value for the character ranged from $56.2(\mathrm{DH} 2752 \times \mathrm{DB} 534)$ to 81.6 $\mathrm{cm}($ DHMS $\times$ SNICB75-10) with a mean of $67.8 \mathrm{~cm}$. Heterosis over mid parent exhibited by hybrids extended from 4.2 (DH2752 $\times$ DB534) to $71.9(\mathrm{RAH}-25-17 \times \mathrm{RAB} 4)$ with a mean of 44.60. All the crosses showed significant positive heterosis over their respective mid-parent. Heterosis values over commercial check ranged from 22.1 (DH2752 $\times$ DB534) to $77.4($ DHMS $\times$ SNICB75-10) per cent with an overall mean of 47.3. All the hybrids exhibited significant positive heterosis. Significant positive heterosis over mid parent was reported by Mallikarjun (2005), Somashekhar (2006), Ramakrishna (2008) and Nidagundi (2010).
The range observed for inter branch distance in hirsutum lines was from $6.5 \mathrm{~cm}$ (DH2752 and RAH 5-10) to $10.5 \mathrm{~cm}$ (RAH 370) with a mean of $8.2 \mathrm{~cm}$ and in barbadense lines this trait varied from $7.5 \mathrm{~cm}$ (RAB 8) to 11 (SNICB 75-10) with a mean of $9.6 \mathrm{~cm}$. The mean value for the trait varied from $7.7 \mathrm{~cm}$ in DHMS $\times$ RAB-4 to $10.4 \mathrm{~cm}$ in $\mathrm{DH} 2752 \times$ RAB8 with an overall mean of $8.9 \mathrm{~cm}$. The hybrids RAH-370 $\times$ SNICB75-10 and DH2752 $\times$ RAB8 showed the maximum mid parent heterosis in negative (-20.9) and positive (48.6) direction, respectively. The mean heterosis was 1.4. Four crosses showed significant positive and six hybrids showed significant negative heterosis over mid parent. With respect to heterosis over commercial check, two crosses recorded significant positive and five crosses recorded significant negative values. Maximum heterosis (14.9\%) was registered in $\mathrm{DH} 2752 \times \mathrm{RAB} 8$ and the minimum $(-14.9 \%)$ was registered by the cross DHMS $\times$ RAB -4 . The mean heterosis was -1.4 per cent.

The range observed for ginning outturn among hirsutum line and barbadense was 31.1 (RAH 5-10) to 37.1 (DHMS) per cent with a mean of 34.5 per cent, whereas in barbadense lines the range was 31.9 (RAB 4) per cent to 33.5 (DB534) per cent having the mean value of 32.8 per cent. The mean range for this trait among the crosses was from 30.7 $(\mathrm{DH} 2752 \times$ DB534) to 41.6 per cent $(\mathrm{RAH}-$ $13-86 \times$ RAB-4) with a mean of 33.9 per cent. The crosses RAH-25-17 $\times$ SNICB75-10 and RAH-13-86 × RAB-4 showed minimum ($11.60 \%)$ and maximum $(24.6 \%)$ mid parent heterosis, respectively. The mean heterosis was 0.8. Twelve crosses showed significant positive heterosis. In case of heterosis over commercial check, nine crosses exhibited significant positive heterosis and the range was from $-6.1(\mathrm{DH} 2752 \times \mathrm{DB} 534)$ to 27.4 $(\mathrm{RAH}-13-86 \times \mathrm{RAB}-4)$ per cent with a mean of 3.8 per cent. 
The range observed for SPAD meter reading was from 41.5 (VB37) to 47.5 (RAH 370) in hirsutum lines with a mean of 44.3 and with a range from 44 (RAB 4) to 49 (SNICB 75-10) with a mean of 45.3 in barbadense lines. The hybrid DHMS $\times$ RAB-4 recorded the lowest mean value (40.0) and DHMS $\times$ SNICB75-10 recorded the highest mean value (52.6) and the overall mean for the character was 43.7.Mid parent heterosis among hybrids ranged from -13.7 $($ RAH-25-17 $\times$ SNICB75$10)$ to $20.1(\mathrm{RAH}-13-86 \times \mathrm{RAB}-4)$ with an overall mean of -2.2 . Two crosses expressed significant positive heterosis while five crosses expressed significant positive heterosis over mid parent. The range of standard heterosis was from -6.9 (RAH-13-86 $\times$ SNICB75-10) to $16.6($ DHMS $\times$ SNICB7510) per cent with a mean of 4.0. Two crosses exhibited significant positive heterosis over commercial check.

Lower range for lint index was observed in both hirsutum (4.5 gm to 6.2gm) and barbadense (4.8 $\mathrm{gm}$ to $7.8 \mathrm{gm}$ ). The lowest and highest lint index was observed in the hirsutum lines of RAH 5-10 and RAH 13-86. Variation for the trait among the crosses was from $5.6(\mathrm{DH} 2752 \times \mathrm{DB} 534)$ to $9.5(\mathrm{RAH}-$ $13-86 \times \mathrm{RAB}-4)$ with an overall mean of 6.8 . The mid parent heterosis among crosses ranged from $3.7(\mathrm{DH} 2752 \times \mathrm{DB} 534)$ to 81 $($ DHMS $\times$ SNICB75-10) with a mean of 31.9. Twenty four crosses exhibited significant heterosis over mid-parent. The crosses DH2752 $\times$ DB534 and RAH-13-86 × RAB-4 recorded minimum (-0.9\%) and maximum $(68.8 \%)$ heterosis values over commercial check, respectively. The mean heterosis was 0.9 , fifteen crosses were noticed to have significant heterosis over commercial check. Presence of positive heterosis over mid parent was reported by Karande et al., (2004). Presence of low heterosis over commercial check for this trait was reported by Maisuria et al., (2006) and Yanal (2013).
Similar to lint index lower range of values for seed index was observed in both hirsutum (8.7 to $11.6 \mathrm{gm})$ and barbadense lines $(9.7 \mathrm{gm}$ to $11.8 \mathrm{gm}$ ) with a mean of $9.7 \mathrm{gm}$ and 10.8 gm respectively. The lowest and highest seed index was observed in the hirsutum line of RAH 16 (8.7gm) and barbadense line RAB $4(11.8 \mathrm{gm})$ respectively. Variation for the trait among the crosses was from 12.3 (DH2752 $\times$ SNICB75-10) to $15.8(\mathrm{VB}-37 \times$ SNICB7510) with an overall mean of 13.2. The mid parent heterosis among crosses ranged from $9.9(\mathrm{RAH}-13-86 \times$ DB534) to $53.70(\mathrm{DH}-37 \times$ SNICB75-10) with a mean of 28.5.

Twenty four crosses exhibited significant negative heterosis. The crosses DHMS $\times$ RAB-4 and DH-37 $\times$ SNICB75-10 recorded minimum $(2.1 \%)$ and maximum $(31.3 \%)$ heterosis values over commercial check, respectively. The mean heterosis was -9.6 and only four crosses were noticed to have significant positive heterosis over commercial check. Similar kind of positive heterosis over mid parent and commercial check was also narrated by Kajjidoni (1982), Reddy (2001), Neelima (2002), Maisuria et al., (2006) and Pole et al., (2008) and Pranesh (2014).

\section{Predicted double cross performance}

The potentiality of thirty two inter heterotic group crosses was assessed and identified top five heterotic boxes based on the principal of predicted double cross performance given by Jenkins (1934). It is important to note that the predicted double cross combination DHMS $\times$ SNICB75-10 and DH2752 × DB534 recorded the highest mean seed cotton yield of $3287 \mathrm{~kg}$ per ha followed by RAH-13-86 $\times$ SNICB7510 and DHMS $\times$ RAB-4 (2841 kg/ha), RAH25-17 $\times$ RAB8 and DH-37 $\times$ DB534 (2753 $\mathrm{kg} / \mathrm{ha}), \mathrm{RAH}-16 \times \mathrm{RAB}-4$ and RAH-370 $\times$ SNICB75-10 (2411 kg/ha) and RAH-5-10 $\times$ RAB4 and RAH-370 × DB534 $(2270 \mathrm{~kg} / \mathrm{ha})$ (Table 3). 
Present study aimed at exploiting heterotic group of hirsutum and barbadense genotypes by forming heterotic box and subjecting it to reciprocal recurrent selection for combining ability. In this study a set of eight genotypes from hirsutum heterotic group crossed with a set of four genotypes from barbadense heterotic group by following line $x$ tester analysis.

Thirty two $\mathrm{F}_{1}$ 's and their parents were evaluated along with check RAHB 87 and DCH 32 at Dharwad to estimate the predicted double cross combination performance of the hybrids for seed cotton yield ( $\mathrm{kg} / \mathrm{ha}$ ) and to identify the heterotic box involving elite lines from opposite heterotic group and for further confirmation of potentiality of the heterotic box chosen for exploitation (through Line $x$ tester analysis).

From the results of the heterosis estimates among the interspecific hybrids, three top hybrids viz., DHMS $\times$ SNICB75-10 (110.2), DH2752 $\times$ DB534 (95.8), DHMS $\times$ DB534 (94.4), RAH-25-17 × DB534 (90.9) and DH2752 × SNICB75-10 (89.4)were identified based on the highest heterosis expression over commercial check in respect of seed cotton yield needs to be verified for their performance on large scale basis.

Based on the predicted double cross performance, the cross combination DHMS $\times$ SNICB75-10 and DH2752 $\times$ DB534 recorded the highest mean seed cotton yield of $3287 \mathrm{~kg}$ per ha. Since the double cross was revealing high potentiality it could be an indication of differences in the dominant favourable alleles distributed among the two single cross parents are different. These viewpoints were kept in mind while selecting the single crosses DHMS $\times$ SNICB75-10 and DH2752 $\times$ DB534 as diverse $F_{1}$ base populations for initiating next phase of reciprocal selection for combining ability.

\section{References}

Bhatade, S. S., V. G. Reddy, S.R. Rajeswar and N. R. Nadre (1992). Diallel analysis of combining ability in certain interspecific crosses of $G$. hirsutum L. J. Indian Soc. Cotton Improv., 17: 26-32.

Deepakbabu, H. (2007). Evaluation of recombinational variability for combining ability in F5 generation of cotton (Gossipium hirsutum L.). M. Sc. (Agri.) Thesis, Univ. Agric. Sci., Dharwad, Karnataka (India).

Deosarkar, D. B., D. S. Jadhav and S. G. Patil (2009). Heterosis study in cotton (Gossypium hirsutum L.) under rainfed conditions. J. otton Res. Dev., 23(1): 36-40.

Dhaka, Bangladesh June 18- 20.Pranesh, K. J. (2014). Exploiting heterotic groups through reciprocal recurrent selection for combing ability to improve performance of cotton hybrids. Ph. D. Thesis, Univ. Agric. Sci, Dharwad, Karnataka.

Falconer, D. S. (1981). Introduction of Quantitative Genetics, Longman Inc. Ltd., New York.

Jenkins, M. T., 1934, Methods of estimating the performance of double crosses in corn. J. American Soc. Agron., 26: 199204.

Kajjidoni, S. T. (1982). Heterosis, combining ability and gene action for earliness, yield and yield components in $2 \times 10$ crosses of $\mathrm{G}$. arboreum $\times \mathrm{G}$. herbaceum cotton. M. Sc. (Agri.) Thesis, Univ. Agric. Sci., Bangalore, Karnataka (India).

Karande, S. S., M. R. Wandhare, M. Y. Ladole, M. M. Waode and L. D. Meshram (2004). Heterosis and combining ability studies in interspecific diploid cotton hybrids for fibre quality parameters. Int. Symp. on Strategies Sustainable Cotton 
Production - A Global Vision 1. Crop Improv., 23-25 November, 2004, Univ. Agric. Sci., Dharwad (Karnataka), India.

Lavanyakumar, K. R. (2004). Genetic studies on plant types of cotton (Gossypium hirsutum L.). M. Sc. (Agri.) Thesis, Univ. Agric. Sci., Dharwad, Karnataka (India). Maisuria, A. T., J. C. Patel, K. G. Patel and B. G. Solanki (2004).

Mallikarjun, H. B. (2005). Evaluation of recombinational variability for combining ability in cotton. Ph. D, Thesis, Univ. Agric. Sci., Dharwad, Karnataka.

Melchinger, A. E. and R. K. Gumber (1998). Overview of heterosis and heterotic groups in agronomic crops. In: Lamkey K. R.

N. G. Ranga Agric. Univ., Hyderabad. Saifullah, A., L. Sawan, Z. A. Deho and M. A. Manjh (2014). Toestimates heterosis and heterobeltosis of yield and qualitytraits in upland cotton. J. Bio. Agril. and Healthcare, 4(6): 2224-2231.

Neelima, S. (2002). Heterosis and combining ability analysis for yield and yield components in cotton (Gossypium hirsutum L.). M. Sc. (Agri.) Thesis, Acharya N. G. Ranga Agric. Univ. Rajendranagar, Hyderabad (India).

Nidagundi, J. M. (2010). Genetic studies on productivity and quality features in cotton (G. hirsutum L.). Ph. D. Thesis, Univ. Agric. Sci, Dharwad, Karnataka (India).

Patil, S. S., V. Ramakrishna, S. M. Manjula, P. Swati, H. M. Ranganatha, H. G. Kencharaddi and H. Deepakbabu (2011). Deploying reciprocal selection for combining ability for improving performance of hybrids in cotton $(G$. hirsutum). Indian J. Genet., 71(2): 180184.

Pole, S. P., S. M. Sudewad, S. K. Kamble and S. B. Borgaonkar (2008). Heterosis for seed cotton yield and yield components in upland cotton (Gossypium hirsutum L.). J.Cotton Res. Dev., 22(2): 139-142.

Potdukhe, N. R. (2002). Level of heterosis for quantitative traits in upland cotton. J. Indian Soc. Cotton Improv., 27(3) :200214

Pranesh, K. J., S. S. Patil, S. M. Manjula, H. G. Kencharaddi and S. Rajeev (2014). Exploitation of heterotic group through reciprocal selection for combining ability in cotton (Gossypium hirsutm L.) paper presented in 6th Meeting of 332 H. G. Kencharaddi et al., the Asian Cotton Research and Developmnet Network,

Punitha, D. and R. Ravikesavan (2004). Heterotic studies in cytoplasmic male sterile based cotton hybrids. PKV Res. J., 28(2): 219-221.

Rajeev, S. (2011). Relating phenotyping and molecular diversity with heterosis in cotton (Gossypium hirsutum). M. Sc (Agri.) Thesis, Univ. Agric. Sci., Dharwad, Karnataka (India).

Rama, Khrishna (2008). Reciprocal selection for combining ability in cotton Ph. D. Thesis, Univ. Agric. Sci, Dharwad, Karnataka.

Reddy, A. N. (2001). Heterosis, combining ability and stability analysis of hybrids for yield and yield components in cotton (Gossypium hirsutum L.). Ph. D. Thesis, Acharya

Shanmugavalli, N. and L. D. Vijendradas (1995). Line $\times$ tester analysis of combining ability in cotton. Madras Agric. J., 82: 449-452.

Somashekhar, D. (2006). Genetic studies on reciprocal selection for combining ability to improve hybrid performance in cotton. Ph. D Thesis, Univ. Agric. Sci., Dharwad, Karnataka (India).

Staub J. E (ed.) Concepts and breeding of heterosis in crop plants. CSSA, Madison, WI. p. 29-44. 
Study of best per se performance, heterosis and combining ability effects for seed cotton yield and its component characters through GMS system in Asiatic cotton. J. Indian Soc. Cotton Improv., 3: 88-91.

Tuteja, O. P. and M. Agrawal (2014). Heterosis for seed cotton yield and other traits in GMS based hybrids of American cotton (Gossypium hirsutum). Cotton Res. J., 5(2): 131-141.

Verma, S. K., S. L. Ahuja, O. P. Tuteja and D. Monga (2004). Line $\times$ tester analysis of yield, its components and fibre quality traits in cotton. J. Indian Soc. Cotton Improv., 29(3): 151-157.

Yanal, A. A. (2013). Development and exploitation of Heteroticpools of $G$. hirsutum and $G$. barbadense for developing potential interspecific hybrids, molecular marker and transformation study in cotton. Ph. D. Thesis, Univ. Agric. Sci, Dharwad, Karnataka.

\section{How to cite this article:}

Rajeev, S., S.S. Patil, S.M. Manjula, K.J. Pranesh, P. Srivalli and Kencharaddi, H.G. 2018. Studies on Heterosis in Cotton Interspecific Heterotic Group Hybrids (G.hirsutum X G.barbadense) for Seed Cotton Yield and Its Components. Int.J.Curr.Microbiol.App.Sci. 7(10): 3437-3451. doi: https://doi.org/10.20546/ijcmas.2018.710.399 